\title{
X-ray Diffraction from X-ray Waveguide Arrays for Generation of Coherent X-ray
}

\author{
Yong-Sung Park and Jaeho Choi* \\ Department of Physics, Dankook University, Cheonan 330-714, Korea
}

(Received May 17, 2010 : revised September 20, 2010 : accepted September 20, 2010)

\begin{abstract}
The generation of coherent $\mathrm{x}$-ray beams by using a multi-slit diffraction phenomenon is presented. The mode-confinement conditions in the x-ray waveguide (XWG) needed to obtain single-mode beams are determined. The XWGs are stacked to form an XWG array. The core of the XWG array is used as a slit in an opaque screen, similar to those used for visible light. Diffraction patterns that interfered constructively in the XWG array are investigated based on multi-slit diffraction theory. The irradiance distributions are studied at on observation screen. The FWHM of diffracted x-ray spectra were between $1.67 \times 10^{-4}$ to $3.30 \times 10^{-5}$ radians which lead to a spot-size of a few tens of micrometers on the screen at distance of $1 \mathrm{~m}$. The intensities decrease with increase in the period of the XWG array, i.e. a thicker cladding, due to growth of the higher-order diffraction peaks.
\end{abstract}

Keywords: Waveguide array, Coherent x-ray, Diffraction

OCIS codes : (050.1940) Diffraction; (340.7480) X-rays, soft x-rays, extreme ultraviolet (EUV); (310.2785)

Guided wave applications

\section{INTRODUCTION}

Recent research efforts have focused on the generation of coherent x-rays for use in applications such as microand nano-imaging as well as in micro-diffraction experiments $[1,2]$. A coherent diffraction-imaging technique has been developed by using monochromatic coherent x-ray beams to investigate the nanostructure of materials [3-5]. The flux of x-rays at the exit of the waveguide is efficiently produced by the enhancement of the internal resonant field in the guiding layer [6]. Bongaerts et. al. demonstrated $\mathrm{x}$-ray waveguides with a multi-step index structure having a gap width of $59 \mathrm{~nm}$ [7]. The beam size was predicted to be about $10 \mathrm{~nm}$ within a tapered waveguide [8]. A passive millimeter-wave imaging system that uses a coherent optical beam-forming technique was reported based on the wave arrays [9].

In this paper, we propose a method for the generation of coherent $\mathrm{x}$-ray beams by using x-ray diffraction from nano-multichannels used as $\mathrm{x}$-ray waveguides. Single-mode $\mathrm{x}$-ray waves that propagate within $\mathrm{x}$-ray waveguides serve as an incident source of diffraction. The diffraction patterns of the x-ray beams at the exit of single-mode waveguide arrays are analyzed for coherent $\mathrm{x}$-ray generation.

\section{METHODS}

We considered an array of single-mode $\mathrm{x}$-ray waveguides as a diffraction aperture similar to a multi-slit opaque screen. By doing so, we enhance the intensity of the E-field of the X-ray waveguide to utilize it as a source of monochromatic and coherent $\mathrm{x}$-ray waves and as the diffraction aperture. A schematic of X-ray waveguide arrays and the coordinates used in the numerical calculation is shown in Fig. 1.

The spatial acceptance depends on the cross section of the core of the waveguide when the incident $\mathrm{x}$-ray beam is directly coupled to the waveguide. The refractive index of the core $n_{1}$, the cladding $n_{2}\left(n_{1}>n_{2}\right)$, and the thickness of the core $d$ are used to describe the waveguide parameter $V=\sqrt{n_{1}-n_{2}} k d$, which is used to determine the number of modes in the waveguide. The $V$ value for the x-ray waveguide can be described by using the deviation coefficient, which is a real part of the refractive index $(n=1-\delta$.),

\footnotetext{
*Corresponding author: choi@dankook.ac.kr

Color versions of one or more of the figures in this paper are available online.
} 


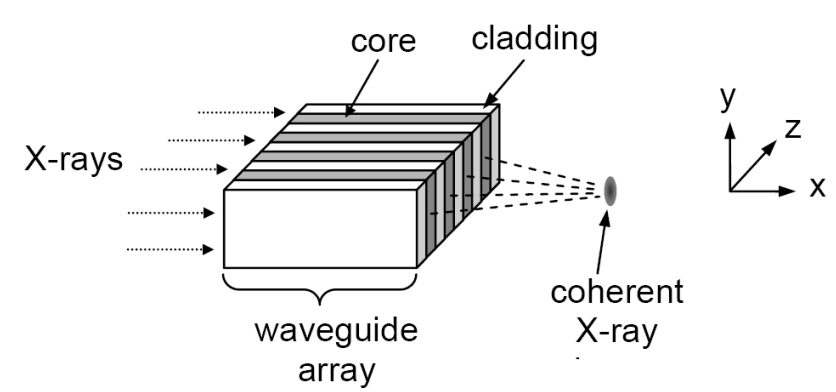

FIG. 1. A schematic of the coordinates for X-ray waveguide arrays.

$$
V \approx \sqrt{2\left(\delta_{2}-\delta_{1}\right)} k d
$$

where the subscripts 1 and 2 denote the core and the cladding, respectively.

The number of modes propagated in a core can be found by using the relationship that $N=[V / \pi]_{\text {int }}$ where $\mathrm{V}$ is the waveguide parameter. The critical thickness of the slab waveguide is given by $d_{c}=\lambda / \sqrt{2\left(\delta_{2}-\delta_{1}\right)}$. When the beam of X-ray waves is incident on the entrance surface, which is perpendicular to the direction of wave propagation in the waveguide, the wave equation for the one-dimensional confinement is independent of the $y$ axis and is given by

$$
\Delta \varphi+k^{2} n^{2}(x, z) \varphi=0
$$

$\varphi$ is written as the product of the function $u(x, y, z)$ and $\exp (-i k x)$ in order to reduce the computing steps in the numerical calculation due to the rapid $x$-direction oscillation of $\varphi$.

$$
\frac{\partial u}{\partial x}=A\left(\frac{\partial^{2} u}{\partial^{2} z}\right)+F(x, z) u
$$

where $u=u_{0} \exp (i k \delta x), A=-i / 2 k, F(x, y, z)=-i / 2 k\left[n^{2}(x, y, z)-1\right]$ The boundary conditions are given as $u(0, z)=u_{0}\left(0, z_{0}\right)$, $u\left(x, z_{0}\right)=u_{0}\left(x, z_{0}\right)$, and $u\left(x, z_{m}\right)=u_{0}\left(x, z_{m}\right)$, where $u_{0}(x, z)$ is an incident-plane wave that is a damping plane wave in the cladding layer. The source code of the numerical calculation is written by using the IDL programming language. When x-ray waves are transmitted through the core, a large number of point sources are spaced evenly across the core of the XWG, the thickness of which is wider than a wavelength. We expect to find maxima and minima in diffracted $x$-ray waves and with the relative phase of these contributions varying by $2 \pi$. The waves leave the exits of the $\mathrm{x}$-ray waveguide array. Those that arrive on the screen in phase produce maxima which preserve the coherency of the single-mode wave.The diffraction patterns of the array of the single-mode XWG are calculated in the same manner as for the multi-slit diffraction of visible light. The irradiance and the resultant amplitude of electromagnetic

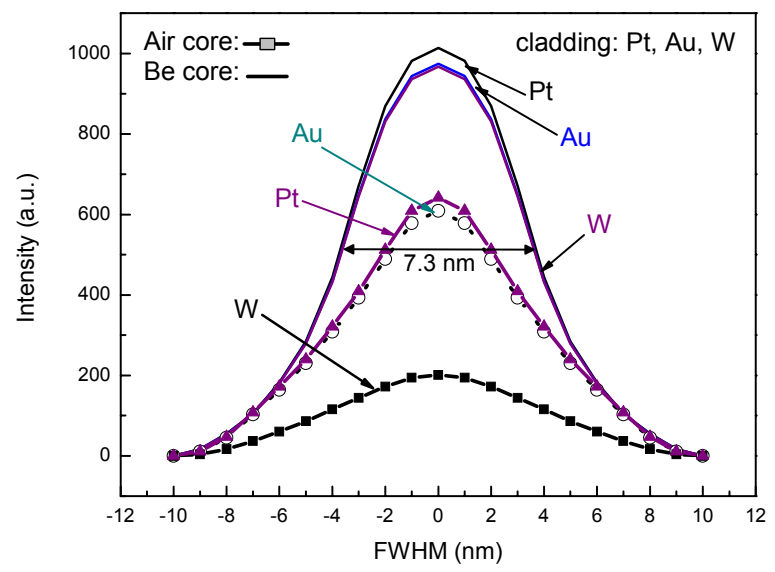

FIG. 2. The single mode profiles at the exit of the XWG for the different claddings.

waves are given by

$$
I=I_{0}\left(\frac{\sin \beta}{\beta}\right)^{2}\left(\frac{\sin N \alpha}{\alpha}\right)^{2},
$$

where $\beta=\pi d \sin \theta / \lambda, \alpha=\pi p \sin \theta / \lambda$ and $p$ is the period of the aperture, which is at a distance equal to the core thickness and one-half of the cladding.

\section{RESULTS AND DISCUSSION}

X-ray waveguides, which confine a beam to a single mode, have been theoretically developed for use as the incident source of the diffraction of X-rays. The array of XWGs consists of up to 20 single-mode XWGs acting as an opaque screen. The slit dimensions are equal to the core thickness of single-mode XWGs (i.e., $16 \mathrm{~nm}$ for an air core and $17 \mathrm{~nm}$ for a beryllium core) which generates coherent $\mathrm{x}$-rays. The deviation coefficient of waveguide materials are $\delta_{1}=5.23 \times 10^{-6}(\mathrm{Be})$ and $\delta_{1}=4.00 \times 10^{-7}$ (air) [10]. The thickness of the tungsten (W) cladding $\left(\delta_{2}=\right.$ $4.61 \times 10^{-5}$ ) varies between 0.25 and 0.5 of the core thickness with $\mathrm{x}$-ray energy of $8.0 \mathrm{keV}$. The field intensities in XWGs with platinum cladding remain higher than those of other cladding materials. However, tungsten has good coupling tendencies, especially with beryllium as the core material.

The single mode profiles at the exit of the XWG array for air and beryllium cores with various cladding materials are shown in Fig. 2 which is a rearrangement of the Fig. 5 and Fig. 6 in Ref. [11]. The optical confinement associated with the normalized propagation constant of its beryllium core XWG is larger than that of its air core. Consequently, the irradiance profile of air core XWGs are far lower than for its beryllium core.

Fig. 3 shows the intensity of the diffracted x-ray spectra in the air-core XWG array. The thickness of the core is 16 
$\mathrm{nm}$ and the cladding thickness is $4 \mathrm{~nm}$. This gives an aperture period of $20 \mathrm{~nm}$, as shown in Fig. 3(a). The diffraction pattern is shown in Fig. 3(b), in which the period of the aperture is increased to $24 \mathrm{~nm}$ while the core thickness remains the same, and the incident x-ray energy is $8.0 \mathrm{keV}$. The full-widths at half maximum (FWHM) were between $1.67 \times 10^{-4}$ radians for $N=4$ and $3.30 \times 10^{-5}$ radians for $N=20$. The FWHM decreases as the number of stacks $N$ in the XWG array increases. The FWHM of the zero-order diffraction peak is about $10 \%$ narrower than the period of $20 \mathrm{~nm}$. However, higher-order diffraction patterns appear with intensity about $20 \%$ of the zero-order diffraction peaks. The FWHM of spectra diffracted from the $17-\mathrm{nm}$ Be core with a period of $21 \mathrm{~nm}$ had values ranging between $1.59 \times 10^{-4}$ radians and $3.18 \times 10^{-5}$ radians. The Be core is thicker than the air core and the FWHM is narrower, possibly because the numerical aperture of the air core is larger than for the $\mathrm{Be}$ core. This is due to the refractive index difference between the cores and the claddings. The FWHM of the coherent spectra after transmission through a $2-\mathrm{cm}$ air gap with a channel thickness $d=500 \mathrm{~nm}$ is $3 \times 10^{-3}$ radians [12].

Our FWHM results for the diffracted spectra from the air-core XWG array is one order of magnitude smaller than values reported in Ref. [12]. From this we can conclude that the XWG array is a device by which it is feasible to generate highly coherent and focusable $\mathrm{x}$-ray beams. The observation that higher-order diffraction patterns appear when the period of the array is increased is also found by calculation. It is explicitly confirmed when compared with the results presented in Ref. [13]. In order to utilize a diffracted x-ray beam as the source of a grating interferometer, the spot size when a zero-order diffraction peak is incident on the screen at $0.1 \mathrm{~m}$ from the XWG array must be calculated, as shown in Fig. 4. The data displayed by the open circles and triangles represent the spot sizes from the air-core XWG array, while the solid lines represent data from the Be core. The spot size
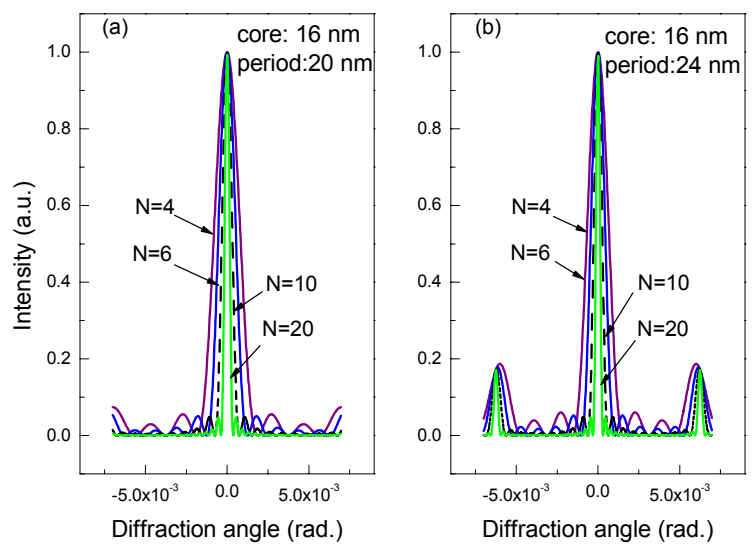

FIG. 3. The intensity of diffracted $x$-ray spectra of the XWG array. on the screen at distance of $0.1 \mathrm{~m}$ from the exit of the XWG array appears smaller for the larger period.

Fig. 5 shows the normalized diffraction intensities at the same distance as in Fig. 4. The intensities decrease with increase in the period of the XWG array. In the Fig. 5 the diffraction intensities from the air core are higher than those from the Be core. These results are obtained under the assumption that the incident photons propagate through the waveguide core without attenuation. However the output intensities from a core vary with different cladding materials as shown in Fig. 2. In particular, the diffractionspectra intensity from the tungsten cladding with air core is only $20 \%$ of that of the W-Be XWG. The results which shows the effect of cladding on the diffraction intensity are inset in Fig. 5. Thus, from the device fabrication point of view, the Pt-, Au-cladding XWG arrays that have the Be-core of $17 \mathrm{~nm}$ with a period of $21 \mathrm{~nm}$ would be best for obtaining the brightest coherent $\mathrm{x}$-rays. The spot size can be tuned by the number of the XWG stacks as well as by the distance of the source to the screen.

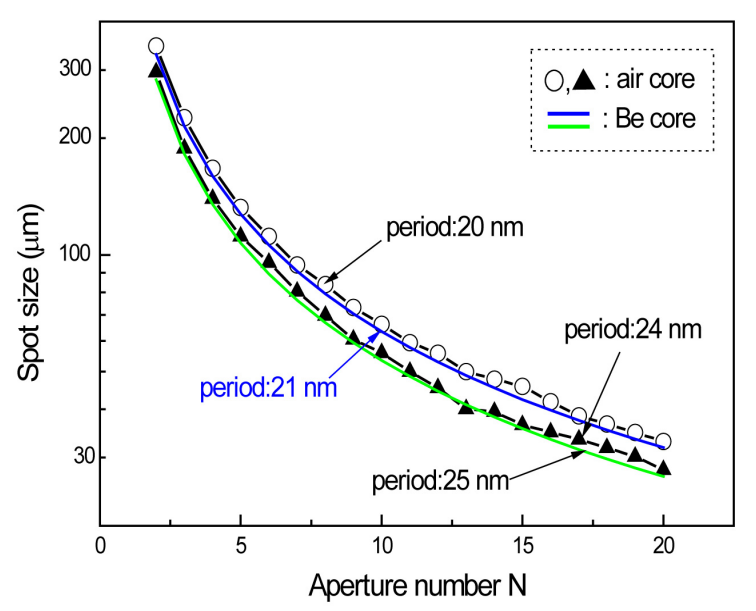

FIG. 4. The spot sizes on the screen at $0.1 \mathrm{~m}$ from the XWG array.

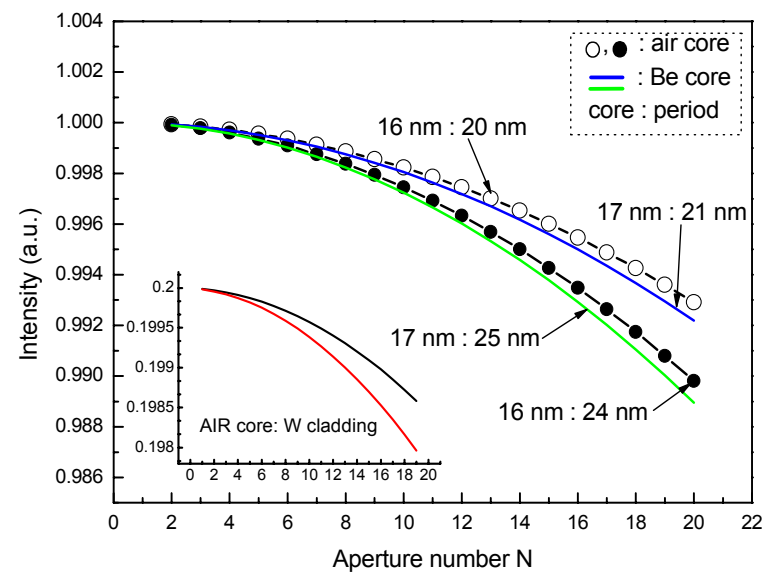

FIG. 5. The normalized intensity of zero-order diffraction-peak at $0.1 \mathrm{~m}$ from the XWG array. 


\section{CONCLUSION}

In this paper we carried out theoretical studies for generation of coherent x-ray beams based on a multi-slit diffraction phenomenon. Numerical calculations were conducted to produce coherent $\mathrm{X}$-ray beams in an XWG for use as a source of multi-slit diffraction. The core of the XWG array is used as the slit of an opaque screen. The FWHM of the diffracted $\mathrm{x}$-ray spectra from the XWG array decreased as the number of stacks $N$ in the XWG array increased.

The intensities of diffracted $\mathrm{x}$-rays decreased for the air core as the aperture period increased to $24 \mathrm{~nm}$ while the core thickness was maintained. For the Be core XWG array, the intensities decreased for the thicker cladding XWG. The FWHM of zero-order diffraction peaks was about $10 \%$ narrower than the period of $20 \mathrm{~nm}$. Higherorder diffraction patterns appear with intensity about $20 \%$ of the zero-order diffraction peaks. The spot size on the screen $0.1 \mathrm{~m}$ from the XWG array had dimensions of a few tens of micrometers. This demonstrates that such x-ray waveguide arrays could be useful for the production of coherent x-ray measurements, such as those used in an $\mathrm{x}$-ray grating interferometer and in a coherent diffraction imaging.

\section{ACKNOWLEDGMENT}

This work was supported by the National Research Foundation of Korea (NRF) grant funded by the Korean government (MEST) (No. 20100020391).

\section{REFERENCES}

1. I. A. Vartanyants, I. K. Robinson, J. D. Onken, M. A. Pfeifer, G. J. Williams, F. Pfeiffer, H. Metzger, Z. Zhong, and G. Bauer, "Coherent x-ray diffraction from quantum dots," Phys. Rev. B 71, 245302-1 245302-9 (2005).

2. J. Miao, Y. Nishino, Y. Kohmura, B. Johnson, C. Song, S. H. Risbud, and T. Ishikawa, "Quantitative image reconstruction of GaN quantum dotsfrom oversampled diffraction intensities alone," Phys. Rev. Lett. 95, 085503-1 085503-4 (2005).

3. S. Marchesini, H. N. Chapman, S. P. Hau-Riege, R. A. London, and A. Szoke, "Coherent x-ray diffraction image: applications and limitations," Opt. Express 11, 2344-2353 (2003).

4. C. Fuhse, C. Ollinger, and T. Salditt, "Waveguide-based off-axis holography with hard x-rays," Phys. Rev. Lett. 97, 254801-1 254801-4 (2006).

5. G. J. Williams, H. M. Quiney, B. B. Dhal, C. Q. Tran, K. A. Nugent, A. G. Peele, D. Paterson, and M. D. de Jonge, "Fresnel coherent diffractive imaging," Phys. Rev. Lett. 97, 025506-1 025506-4 (2006).

6. W. Jark, A. Cedola, S. D. Forzo, M. Fiordelisi, S. Lagomarsino, N. V. Kovalenko, and V. A. Chernov, "High Gsin beam compression in new-generation thin-film x-ray waveguides," Appl. Phys. Lett. 78, 1192-1194 (2001).

7. J. H. H. Bongaerts, M. J. Zwanenbury, F. Zontone, and J. F. van der Veen, "Propagation of coherent $x$-rays in a multistep-index x-ray waveguide," J. Appl. Phys. 90, 94-100 (2001).

8. C. Bergermann, H. Keymeulen, and J. F. van der Veen, "Focusing X-ray beams to nanometer dimensions," Phys. Rev. Lett. 91, 204801-1 204801-4 (2003).

9. P. M. Blancherd, A. H. Greenaway, A. R. Harvey, and K. Webster, "Coherent optical beam forming with passive millimeter-wave arrays," IEEE J. Lightwave Technol. 17, 418-425 (1999).

10. Center for X-ray Optics Web Site: www-cxro.lbl.gov/.

11. J. Choi, J. Jung, and T. Kwon, "Mode propagation in X-ray waveguides," J. Opt. Soc. Korea 12, 112-117 (2008).

12. L. I. Ognev, "X-ray diffraction effects in a submicron slits and channel," X-ray Spectrom. 31, 274-277 (2002).

13. C. Ollinger, C. Fufse, A. Jarre, and T. Salditt, "Two-dimensional X-ray waveguides on a grating," Physica B 357, 53-56 (2005). 